\title{
Estudio de las competencias de los emprendedores/innovadores sociales. El caso del Premio ELI de la Universidad EAN
}

FECHA DE RECEPCIÓN: 27 de julio

FECHA DE APROBACIÓN: 26 de agosto

Pp. 75-90

DOI: http://dx.doi.org/10.21158/01208160.n81.2016.1557

\section{Resumen}

La innovación social y el emprendimiento social se han convertido en alternativas empresariales, indistintamente de si son estas con o sin ánimo de lucro. El presente artículo identifica las competencias del innovador y del emprendedor social a partir del concepto de quienes consideran que hacen parte de estas categorías. La información usada en el proyecto fue recogida entre los participantes del premio ELI, organizado por la Universidad EAN en 2013 y 2014.

\section{Palabras clave}

Emprendimiento social, innovación social, competencias, emprendimiento, innovación.

\section{Omar Alonso Patiño Castro}

PhD. en Ciencias Empresariales, Universidad Antonio de Nebrija; magíster en Gestión de Organizaciones, Universidad du Quebec, Canada; especialista en Gestión de la Calidad y Procesos de Innovación, Universidad Javeriana; administrador de empresas, Universidad Javeriana. Profesor titular de la Universidad EAN.

\section{Edwin Andrés Cruz Pérez}

Magíster(c) en Ciencias-Estadística, Univesidad Nacional de Colombia; especialista en Estadística, Universidad Nacional; licenciado en Matemáticas, Universidad Distrital Francisco José de Caldas. Profesor asistente, Universidad EAN.

\section{Martha Cecilia Gómez Melo}

Magíster en Administración, Universidad Nacional de Colombia; magíster en Direccionamiento Estratégico y Gestión de la Innovación, Universidad Autónoma de Barcelona; Economista, Universidad Nacional de Colombia. Profesora, Universidad EAN. 


\section{Study about the Entrepreneurs' or Social Innovators' Competencies Case Study of the EAN ELI Prize}

Abstrac. Social innovation and entrepreneurship have become managerial alternatives, even if they are used for profit or non-profit purposes. This article aims at identifying the competencies of the social innovator and entrepreneur focusing on the basic concept of those who consider that they belong to those categories. The information used in this project was collected by the ELI Prize participants organized and held at Universidad EAN in 2013 and 2014.

Key words. Social entrepreneurship, social innovation, competencies, entrepreneurship, innovation.

\section{Étude des compétences des entrepreneurs-innovateurs sociaux: le cas du prix ELI de I'Université EAN}

Resumé. L'entrepreneuriat et l'innovation sociale à but lucratif et non lucratif se sont convertis en alternatives entrepreneuriales. Cet article identifie les compétences de l'entrepreneur et innovateur social à partir des concepts de leurs pairs certifiant leur appartenance à ces catégories. Les sources d'informations utilisées dans ce projet ont été rendues publiques par les participants au prix ELI organisé par I'Université EAN en 2013 et 2014.

Mots clefs. Entrepreneuriat social, innovation sociale, compétences, entrepreneuriat, innovation.

\section{Estudo das competências dos empreendedores/inovadores sociais. O Caso do Prêmio ELI da Universidade EAN}

Resumo. A inovação social e o empreendimento social transformaram-se em uma alternativa empresarial, independentemente do objetivo final ser com ou sem fins lucrativos. Este artigo identifica as competências do inovador e do empreendedor social, a partir do conceito de quem considera que faz parte destas categorias. A informação utilizada no projeto foi recolhida pelos participantes do prêmio ELI, organizado pela Universidade EAN em 2013 e 2014.

Palabras chave. Empreendimento social, inovação social, competências do empreendedor social, empreendimento, inovação. 


\section{Introducción}

E I fomento a proyectos de innovación, especialmente cuando estos cuentan con un componente de impacto social, ha sido un propósito que se han fijado organizaciones de todo tipo, desde los gobiernos, a través de entidades creadas para ese fin específico, hasta la empresa privada, pasando por organizaciones de diversa índole, entre las cuales se encuentran las universidades.

En tal sentido, la Universidad EAN concibió, en 2013, el reconocimiento a proyectos diseñados y ejecutados por jóvenes que con su trabajo aportan significativamente a la construcción de un nuevo país

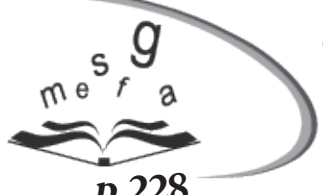

p.228 sostenible desde lo económico, social y ambiental. Como producto de ello, creó el Premio al Emprendimiento, Liderazgo e Innovación (ELI), para cuya realización en ese mismo año, contó con la colaboración del programa Colombia Joven, de la Presidencia de la República y el diario La República; en 2014, lo tuvo con la Casa Editorial El Tiempo a través del diario Portafolio.
Con el Premio ELI, la Universidad EAN ha buscado identificar y reconocer a jóvenes colombianos que han puesto en marcha proyectos que pueden ser considerados como innovadores, desde lo social, orientados al desarrollo de sus comunidades, a la atención y solución de necesidades sociales o a la disminución de la pobreza, mediante ideas creativas en el ofrecimiento de productos o servicios a esas comunidades necesitadas.

Este artículo muestra diferentes interpretaciones de los conceptos de innovación social y emprendimiento social y las relaciones existentes entre ellos. Luego, se presentan los resultados del Premio ELl; $y$, por último, se describen los hallazgos del trabajo de investigación en cuatro elementos básicos: autoevaluación de la innovación del proyecto; aportes recibidos a lo largo del proceso de educación de los emprendedores; motivos para la formulación de proyectos sociales; y determinación de las competencias más importantes para los encuestados y la correlación existentes entre ellas. 


\section{Metodología}

T a investigación que se realizó es un estudio $\checkmark$ observacional descriptivo con metodología cuantitativa, la cual se llevó a cabo mediante un diseño social tipo encuesta transversal, como instrumento aplicado a un tamaño de muestra específico.

El trabajo de campo fue realizado sobre los participantes del concurso. Para el levantamiento de la información estadística se elaboró una encuesta aplicada a los participantes del Premio ELI en sus versiones 2013 y 2014, cuyo total fue de $204-117$ y 87 , respectivamente-.

Para el estudio, se planteó un muestreo aleatorio simple, calculando la muestra con un margen de error de $\varepsilon=5 \%$, nivel de confianza del $95 \%$ que conlleva a un valor cuantil de la distribución normal estándar de $Z=1.96$ y un $p=94 \%$-estimación de la proporción de personas de la población que tienen competencias emprendedoras sociales-, para un tamaño muestral de $\mathrm{n}=61$ personas encuestadas.

En el diseño de la encuesta se proyectaron 47 preguntas, la cual fue presentada a cinco expertos que la validaron de forma cualitativa, teniendo en cuenta su relevancia, coherencia, suficiencia y claridad; de esta manera lograron corroborar el entendimiento de la encuesta e identificaron posibles oportunidades de mejora. El resultado de la validación por expertos permitió realizar el ajuste y corrección de algunas preguntas, las cuales se transformaron y volvieron al formulario.

La recolección de información se realizó de forma virtual, usando formularios de Google Drive, los cuales estuvieron disponibles para diligenciar por Internet. Para el procesamiento de datos se utilizó el software Análisis estadístico SPSS. La significancia de las diferencias entre proporciones, se analizó utilizando pruebas de chi-cuadrado, de Pearson, resultados que se presentarán en un artículo posterior.

\section{Marco teórico}

Dara determinar las competencias asociadas a los emprendedores e innovadores sociales, se partió de una verificación de los

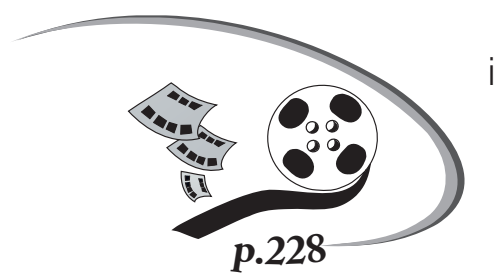

conceptos relevantes de innovación y emprendimiento social, lo que conduce a entender las competencias asociadas en uno y otro caso, como un conjunto interrelacionado con múltiples similitudes. Por ello, la investigación hace referencia a competencias de los emprendedores e innovadores sociales, indistintamente.

\subsection{La innovación social}

Durante el presente siglo, la innovación social ha adquirido un papel fundamental entre las estrategias con las cuales se quiere disminuir la pobreza que afecta a cerca del 9,6\% de la población mundial (Banco Mundial, 2016); como producto de ello, se ha convertido en objeto de estudio con el fin de determinar las condiciones que han hecho que su crecimiento haya sido tan rápido y con impactos significativos en comunidades específicas. 
Innovación social es un término de reciente aparición, pero como concepto tiene diversos antecedentes a lo largo de la historia, incluyendo, para muchos, metodologías y actividades como hospicios, cooperativas o microcrédito y su posterior desarrollo en microfinanzas (Murray, Caulier-Grice y Mulgan, 2010). Sin embargo, el primer gran impulso de la innovación, como una de las estrategias de desarrollo, se dio en el marco del Consejo Europeo Extraordinario de Lisboa, en el que se pidió a los países miembros de la Unión Europea la creación de condiciones favorables para la innovación, el espíritu empresarial y la inversión, con la creación de la Zona Europea de Investigación e Innovación (Consejo Europeo de Lisboa, 2000).

Si bien, la innovación fue concebida por Schumpeter (1911) bajo supuestos económicos, según los cuales un sujeto económico crea, estructura y ofrece al mercado nuevas ideas, productos o servicios, actualmente se considera la innovación social más allá del puro concepto de innovación; no puede contar con una sola definición, y por el contrario, en su concepción multidimensional debe ser entendida como la consecuencia de actividades y procesos que buscan la superación de problemas sociales, pero que en su fondo tienen un amplio sentido de conciencia social.

En la búsqueda de esas múltiples dimensiones, Martínez (2011), plantea que la innovación social debe ser abordada desde cuatro enfoques distintos: el económico, el administrativo, el socioecológico y el político. Desde una perspectiva económica, la innovación social se acerca a la visión de Schumpeter, en la cual se expresa a través de nuevas ideas que satisfacen necesidades sociales originadas por un emprendedor social.
Desde lo administrativo, se plantea que la innovación social es una solución más eficaz, eficiente y sostenible que otras anteriores, con creación de mayor valor público,

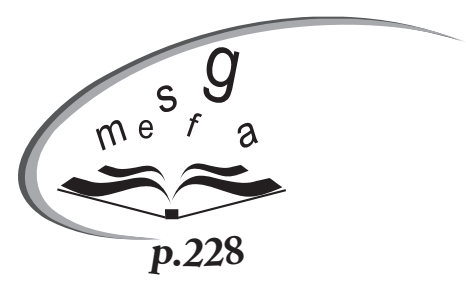
entendido como la cobertura a un mayor número de personas, por encima de la creación de valor desde lo individual.

En el enfoque socioecológico, Martínez (2011), plantea que la innovación social es un proceso complejo sobre comunidades vulnerables, que busca cambiar de manera profunda las rutinas existentes en un sistema social mediante la inclusión de nuevos productos o programas, promovidos por la misma población o por un emprendedor institucional.

Por último, desde la visión política, la innovación social busca la inclusión de sectores desprotegidos en múltiples ámbitos de la sociedad, cambiando la dinámica de sus relaciones y teniendo como origen iniciativas de la misma sociedad.

Independientemente del enfoque asumido, la innovación social proviene de grupos que pueden perseguir distintos fines desde lo personal o empresarial. En este sentido, los proyectos pueden ser impulsados por organizaciones sin ánimo de lucro, por empresas que a través de la satisfacción de las necesidades de determinada comunidad vulnerable ven la oportunidad de generar un negocio lucrativo o, por el mismo Estado a través de programas de promoción a iniciativas particulares o de la creación de agencias especializadas para tal fin. 
En consonancia con lo anterior, Murray, Caulier-Grice y Mulgan (2010), plantean que la innovación social no se refiere a un sector determinado de la economía, sino a la innovación en la creación de productos y resultados sociales independientemente de donde nacen; así, concluyen que esta no puede limitarse a un sector, sino que tienen que cubrirse todos los sectores y la dinámica de sus relaciones.

Tal como se ha planteado, no existe la definición única y exacta de innovación social. Con el fin de dar un contexto, una aproximación cercana a todos los abordajes descritos anteriormente, Abreu (2011), menciona:

\section{Nuevos procesos, prácticas, usos, méto- dos o aplicaciones en relación con el estado del arte de la región en las cuales se desarrolla o fortalece la participación de la comunidad o los beneficiarios, y que permita o tenga el potencial para reducir los costos, incrementar la cober- tura o la calidad, la pertinencia y la eficacia del proyecto.}

De esta forma, dejó circunscrito el significado al ámbito privado, al cual pertenecen los sujetos participantes en este proceso de investigación.
Acorde con lo anterior, la Cepal (2010), ha planteado que las condiciones básicas que conlleva un proyecto de innovación social son: el grado de innovación, su potencial de sostenibilidad y de replicabilidad, los efectos sobre los determinantes de la pobreza en los beneficiarios, el desarrollo de la responsabilidad social en el grupo, el nivel de fomento al trabajo colaborativo y en redes, y el fortalecimiento de las capacidades de liderazgo de los innovadores. Estas condiciones están directamente relacionadas con los innovadores sociales gestores, debido a que ellos son quienes hacen emerger los procesos de desarrollo en sus áreas de influencia.

\subsection{Emprendimiento social}

En el caso del emprendimiento social, existen dos líneas ideológicas bien definidas: la primera, considera que este fenómeno debe ser concebido como una variante del emprendedor, y la segunda, según la cual se cree que el emprendedor social es una categoría aparte, lejana de los emprendedores de negocio. Enciso et al.(2012), realizan una recopilación de definiciones provenientes de distintos autores, en la que se evidencian diversas opiniones y algunos de los argumentos sobre los cuales se basa la dicotomía antes enunciada. A continuación, se presentan algunos de sus aspectos más importantes (Tabla 1): 
Tabla 1. Definiciones de emprendedor social.

\begin{tabular}{|c|c|}
\hline Fuente & Definición \\
\hline Ashoka & $\begin{array}{l}\text { Los emprendedores sociales son individuos con soluciones innovadoras } \\
\text { para los problemas sociales más acuciantes. Son ambiciosos y persistentes, } \\
\text { abordando las principales cuestiones sociales y ofreciendo nuevas ideas para } \\
\text { un cambio a gran escala. Son tanto visionarios como extremadamente realistas, } \\
\text { preocupados por la puesta en práctica de su visión por encima de cualquier otra } \\
\text { cuestión. El emprendedor social presenta ideas que son sencillas para el usuario, } \\
\text { comprensibles, éticas y compromete un apoyo generalizado para maximizar la } \\
\text { cantidad de personas que podrán ponerse en pie, aprovechar su idea y llevarla } \\
\text { a la práctica. }\end{array}$ \\
\hline Echoing Green & $\begin{array}{l}\text { Emprendedores sociales son aquellos individuos excepcionales que sueñan y } \\
\text { aceptan la responsabilidad de una idea innovadora y aún no probada para el } \\
\text { cambio positivo; y acompañan dicha idea desde el sueño hasta la realidad. Lo } \\
\text { que permite a los emprendedores sociales conseguir impacto duradero en los } \\
\text { más difíciles problemas es una combinación especial de creatividad rompedora } \\
\text { y ejecución rotunda, que nosotros denominamos el coeficiente de inteligencia } \\
\text { social emprendedora. }\end{array}$ \\
\hline Schwab Foundation & $\begin{array}{l}\text { Los empresarios sociales pilotan la innovación y transformación social hacia } \\
\text { varios campos, incluyendo educación, salud, medio ambiente y desarrollo } \\
\text { empresarial. Persiguen aliviar la pobreza con celo emprendedor, métodos } \\
\text { empresariales y el coraje de innovar y superar las prácticas tradicionales. }\end{array}$ \\
\hline $\begin{array}{c}\text { Skoll Centre for social } \\
\text { entrepreneurship }\end{array}$ & $\begin{array}{l}\text { Los emprendedores sociales son agentes de cambio en la sociedad, creadores } \\
\text { de innovaciones que trastocan el statu quo y transforman nuestro mundo a } \\
\text { uno mejor. }\end{array}$ \\
\hline
\end{tabular}

Fuente. Elaboración propia de los autores a partir de Enciso et al. (2012).

De lo anterior se resalta la coincidencia existente en algunas de las condiciones del emprendedor social, entre ellas: una buena idea, creatividad, potencial para extender el impacto, calidad empresarial y fuerte fibra ética.

Es claro que al hablar de emprendimiento social, las características se mezclan con la innovación social. De hecho aparecen con frecuencia

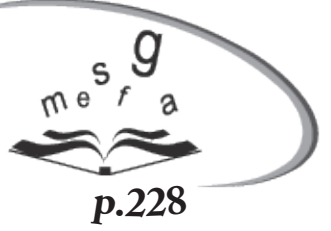
remitiéndose a tres componentes básicos propios de la innovación: respuesta a fallos en el mercado-problemas sociales-, innovación transformadora y sostenibilidad financiera.

Para López (2014), existe un elemento presente en todas las definiciones de emprendimiento social, y radica en que tiene un doble objetivo social: por una parte, busca solucionar problemas sociales, y por la otra, está unido a la sostenibilidad de la empresa. Este elemento se suma a que debe contener un alto impacto en la sociedad.

En cuanto al emprendedor social, Sastre (2014), refiere algunas de las competencias planteadas por otros autores: alto nivel de compromiso, fortaleza ante la adversidad, capacidad para asumir riesgos, capacidad de generar confianza y credibilidad en terceros (Thomson, Alvy \& Lees, 2000); creatividad, valentía y fortaleza ante las dificultades, alto grado de compromiso, capacidad para asumir riesgos (Sullivan et al., 2003); sensibilidad a la exclusión, la marginación o el sufrimiento de aquellos que carecen de medios económicos o influencia política (Martin y Osberg, 2007); 
amabilidad, abierto a las ideas, autoexigencia en el trabajo (Nga \& Shamuganathan, 2010); y carisma y fe inquebrantable en el proyecto (Jiao, 2011).

Para Dess (2010), las claves para identificar a un emprendedor social se encuentran en tres aspectos: a) la misión que tiene que ver con solucionar un problema social que no riñe con la generación de ingresos; b) la innovación, ya que debe ofrecer soluciones creativas y diferentes, y c) los ingresos: la empresa social no tiene por qué eludir la financiación a través de la generación de ingresos y beneficios propios. Los recursos generados facilitarán la supervivencia y el éxito de la empresa social, lo que ayudará a cubrir las necesidades de grupos socialmente excluidos (Riddley-Duff, 2008) y aumentará el bienestar social (Murphy \& Coombes, 2009), ganando, a su vez, legitimidad ante los posibles donantes.

\section{Las competencias de los emprendedores/ innovadores sociales}

$\mathrm{R}$ evisando los conceptos de innovación y emprendimiento social, se encuentran grandes similitudes, por lo cual se consideran las competencias requeridas para desarrollar uno y otro, como un conjunto de habilidades relacionadas profundamente con las características para ejecutar los proyectos que consigan un impacto en la generación de ingresos y cuya utilidad se redistribuya socialmente. Tal vez, la única diferencia sustancial es el énfasis en la generación de ingresos del emprendedor, aspecto no desestimado, pero tampoco trascendental para el desarrollo del innovador social.

Una competencia se define desde las nuevas teorías de la cognición, básicamente como:

Saberes de ejecución. Puesto que todo proceso de conocer se traduce en un saber, entonces es posible decir que son recíprocos competencia y saber: saber pensar, saber desempeñar, saber interpretar, saber actuar en diferentes escenarios, desde sí y para los demás (dentro de un contexto determinado) Vásquez (2009).
Para Gilomen (2006), las competencias claves deben contribuir a que los ciudadanos lleven una vida de éxito y buen funcionamiento de la sociedad.

McClelland (1973), además de definir el término competencia, expandió su uso disciplinar, haciendo referencia al enfoque de recursos humanos orientados a mejorar los procesos productivos; sin embargo, la evolución del concepto lleva hoy a pensarlas dentro de variados contextos, incluso del emprendimiento y la innovación. En esta dimensión, las competencias emprendedoras «son aquellas que le permiten a los sujetos desarrollar un proyecto emprendedor con el que generan crecimiento económico y cohesión social, configurándose así como un proyecto social integrado» (Martínez, 2009).

Acorde con lo anterior, el mismo autor considera que las competencias emprendedoras deben facilitar el éxito de la iniciativa, y de la misma manera, ayudar en el cultivo de «valores y prácticas sociales asentadas en principios democráticos...» (Martínez, 2009) (Tabla 2). 
Tabla 2. Definiciones de competencias emprendedoras.

\begin{tabular}{|c|l|}
\hline Organismo & \multicolumn{1}{c|}{ Definición } \\
\hline OCDE (2005) & $\begin{array}{l}\text { La denomina competencia en autonomía, y consiste en: capacidad de los } \\
\text { individuos de controlar su vida de forma responsable y con sentido, ejerciendo un } \\
\text { grado de control sobre sus condiciones de vida y trabajo. La acción autónoma es } \\
\text { necesaria para participar eficazmente en la sociedad; incluye tres competencias } \\
\text { clave: capacidad de actuar en el marco general, capacidad de formar y llevar a } \\
\text { cabo planes de vida y proyectos personales, y habilidad para definir los derechos e } \\
\text { intereses, límites y necesidades. }\end{array}$ \\
\hline Unión Europea & $\begin{array}{l}\text { Define el espíritu emprendedor como: la capacidad para provocar uno mismo } \\
\text { cambios-componenteactivo-yhabilidad para aceptaryapoyarcambios producidos } \\
\text { por factores externos -componente pasivo-. Incluye la aceptación del cambio, } \\
\text { asumiendo la responsabilidad de las propias acciones -positivas o negativas- } \\
\text { marcando objetivos y alcanzándolos, teniendo motivación para alcanzar el éxito. } \\
\text { Conlleva el conocimiento de oportunidades existentes con el fin de identificar las } \\
\text { más adecuadas para los propios proyectos personales, profesionales o negocios. }\end{array}$ \\
\hline RINACE (2009) & $\begin{array}{l}\text { Las competencias emprendedoras buscan la autonomía de la persona y se } \\
\text { orientan hacia la autorrealización del sujeto, como vía para la realización de un } \\
\text { proyecto vital centrado en la producción de bienes y servicios que satisfagan las } \\
\text { necesidades de la comunidad. Los individuos son concebidos como objeto y sujeto } \\
\text { de la transformación, que da lugar a la reactivación del sistema productivo y el } \\
\text { incremento de la actividad económica de sus regiones de origen. }\end{array}$ \\
\hline
\end{tabular}

Fuente. Elaboración propia de los autores a partir de Martínez (2009).

Otros autores, Christersen (1994), Olamendi (2005) y Domínguez (2004), plantean como parte de las competencias emprendedoras/ innovadoras sociales, las siguientes: actitud mental positiva, liderazgo y capacidad para delegar, gestión del tiempo y planificación, capacidad de negociación, capacidad para sobreponerse al fracaso, actitud ética y proactiva, iniciativa facilidad para entablar relaciones sociales para trabajar bajo presión. 


\section{Hallazgos}

a población que participó en el estudio tuvo una composición de $62,30 \%$ por hombres, y un $37,70 \%$ por mujeres, mientras que por nivel educativo se distribuyó de la siguiente manera: el $23 \%$ tienen estudio de secundaria, el 68,8 \% estudios universitarios, y el $8,2 \%$ estudios de posgrado.

A pesar de que un $96,7 \%$ de las personas consideran que han realizado proyectos de emprendimiento social, esta cifra se reduce a un $88,5 \%$ cuando se pregunta si se considera que los proyectos han sido innovadores. En cuanto al aporte que estos emprendedores han recibido en sus procesos académicos en el colegio o la universidad, apenas un 44,3\% señalaron que las enseñanzas obtenidas en estas instituciones les han contribuido en el diseño de sus proyectos de emprendimiento.

En la pregunta: ¿Para usted, emprendimiento social es? A los encuestados se le pidió ordenar las distintas definiciones de la siguiente forma: el elemento de mayor prioridad debe estar en la parte superior, mientras el menos importante debe estar en la posición más baja. Las alternativas dadas al encuestado fueron las siguientes:

- Ayudar a la sociedad sin interés económico.

- Buscar soluciones empresariales a problemas sociales.

- Crear valor social sostenible a partir de iniciativas empresariales.
- Generar soluciones innovadoras a problemas graves de la sociedad.

- Satisfacer las necesidades de la sociedad.

Para poder hacer un análisis del ítem, se asignó una respuesta numérica de 1 a 5 , según el orden en que organizó las definiciones cada uno de los encuestados (Figura 1).

Acorde con lo anterior, se puede evidenciar, que, a pesar de que hay valores semejante para todas las alternativas, el motivo mejor valorado por los emprendedores para hacer emprendimiento social es: generar soluciones innovadoras a problemas graves de la sociedad, con una moda de 1, siendo 1 la máxima puntuación en la escala; y el factor menos importante es crear valor social sostenible a partir de iniciativas empresariales, el cual alcanza un valor modal de 5 teniendo las otras alternativas una distribución uniforme por los rangos de respuesta.

Para la pregunta: ¿Su interés para la realización de estos proyectos ha sido? Se solicitaba a los encuestados marcar las entradas que correspondan señalando una o más de las siguientes opciones planteadas: identificación de oportunidades y opción de negocio; proyecto de vida al servicio de la sociedad; problemas propios, de su familia o de la comunidad; ejercicio de la responsabilidad social; y muchas personas lo hacen y la sociedad lo necesita (Tabla 3). 
Figura 1. Motivos del emprendedor para el emprendimiento social.

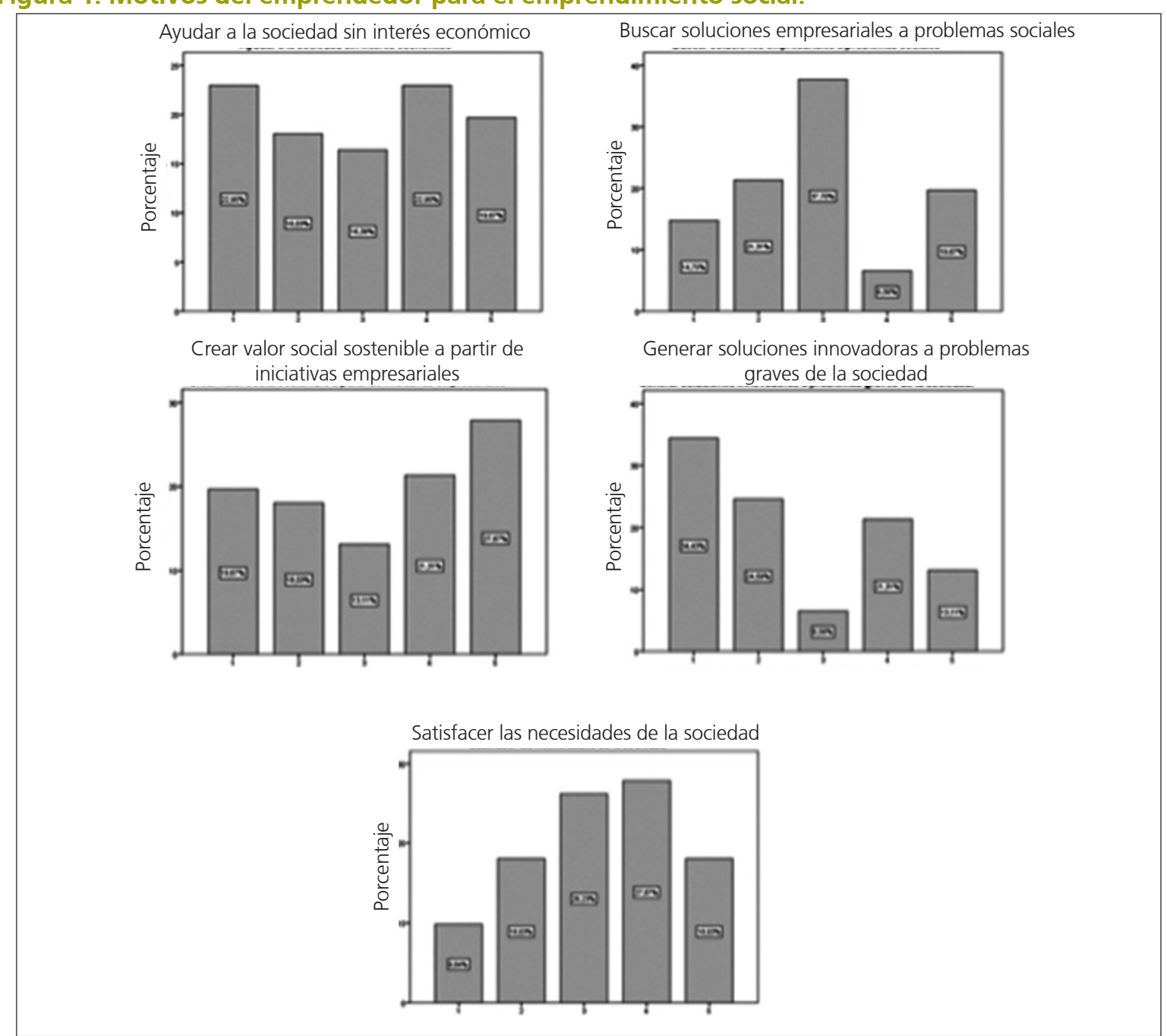

Fuente. Elaboración propia de los autores.

Tabla 3. Interés para la realización de proyectos de emprendimiento social.

\begin{tabular}{|c|c|c|c|c|}
\hline & \multicolumn{2}{|c|}{ Respuestas } & \multirow{2}{*}{$\begin{array}{l}\text { Porcentaje } \\
\text { de casos }\end{array}$} \\
\hline & & $\bar{n}$ & Porcentaje & \\
\hline \multirow{6}{*}{$\begin{array}{l}\text { Su interés para la } \\
\text { realización de estos } \\
\text { proyectos ha sido? }\end{array}$} & $\begin{array}{l}\text { Identificación de oportunidades y opción } \\
\text { de negocio. }\end{array}$ & 35 & $22,8 \%$ & $57,4 \%$ \\
\hline & Proyecto de vida al servicio de la sociedad. & 42 & $26,8 \%$ & $68,9 \%$ \\
\hline & $\begin{array}{l}\text { Problemas propios, de su familia o de la } \\
\text { comunidad. }\end{array}$ & 32 & $20,4 \%$ & $52,5 \%$ \\
\hline & Ejercicio de la responsabilidad social. & 40 & $25,5 \%$ & $65,6 \%$ \\
\hline & $\begin{array}{l}\text { Muchas personas lo hacen y la sociedad lo } \\
\text { necesita. }\end{array}$ & 8 & $5,1 \%$ & $13,1 \%$ \\
\hline & Total & 157 & $100,0 \%$ & $257,4 \%$ \\
\hline
\end{tabular}

Fuente. Elaboración propia de los autores. 
Teniendo en cuenta que la muestra era de 61 personas, hubo en total 157 marcaciones dado que cada encuestado podía seleccionar una o más opciones. Se destacan los ítems Proyecto de vida al servicio de la sociedad, y Ejercicio de la responsabilidad social con un $26,8 \%$ y $25,5 \%$, respectivamente, siendo estos los porcentajes más altos. Los encuestados mostraron poco interés por el item Muchas personas lo hacen y la sociedad lo necesita, el cual ponderó un 5,1 \% de las respuestas totales.
De la misma forma que la pregunta anterior, el participante podía marcar una o más opciones al ítem ¿Cuáles han sido sus principales logros en el proyecto(s) de emprendimiento social? Las opciones corresponden a (Tabla 4):

- Impacto social.

- Beneficio económico.

- Reconocimiento en la comunidad.

- Consecución de recursos para la empresa.

Tabla 4. Logros obtenidos en los proyectos de emprendimiento social

\begin{tabular}{|c|c|c|c|c|}
\hline & \multicolumn{2}{|c|}{ Respuestas } & \multirow{2}{*}{$\begin{array}{c}\text { Porcentaje } \\
\text { de casos }\end{array}$} \\
\hline & & $\bar{n}$ & Porcentaje & \\
\hline \multirow{4}{*}{$\begin{array}{l}\text { ¿Cuáles han sido sus } \\
\text { principales logros en } \\
\text { el proyecto(s) de } \\
\text { emprendimiento } \\
\text { social? }\end{array}$} & Impacto social. & 56 & $38,4 \%$ & $91,8 \%$ \\
\hline & Beneficio económico. & 31 & $21,2 \%$ & $50,8 \%$ \\
\hline & $\begin{array}{l}\text { Reconocimiento en la } \\
\text { comunidad. }\end{array}$ & 43 & $29,5 \%$ & $70,5 \%$ \\
\hline & $\begin{array}{l}\text { Consecución de recursos } \\
\text { para la empresa. }\end{array}$ & 16 & $11,0 \%$ & $26,2 \%$ \\
\hline \multicolumn{2}{|r|}{ Total } & 146 & $100,0 \%$ & $239,3 \%$ \\
\hline
\end{tabular}

Fuente. Elaboración propia de los autores.

Se evidencia, que para la mayoría de los encuestados, el principal logro en los proyectos de emprendimiento tiene que ver con el impacto social, con un 38,4 \%; por otro lado, la consecución de recursos para la empresa y el beneficio económico, con $11 \%$ y $21,2 \%$, respectivamente; se puede observar, que la prioridad en los logros está dado por el impacto y el reconocimiento social, mas no por los beneficios económicos.

A la pregunta ¿Cuáles han sido sus principales obstáculos en el proyecto(s) de emprendimiento social?, el 31,1 \% de los encuestados cree que el principal obstáculo es el apoyo institucional seguido de la falta de recursos, con un 26,4 \% (Tabla 5).
En la segunda parte del cuestionario, se le pedía al encuestado evaluar la importancia de ocho características que debe tener un emprendedor, en una escala de 1 al 5, donde cinco representa la valoración más alta. Las características por evaluar eran las siguientes:

- Innovador.

- Persistente.

- Consciente de los problemas sociales.

- Convincente.

- Vendedor.

- Líder.

- Sensitivo.

- Proactivo. 
Tabla 5. Obstáculos afrontados en los proyectos de emprendimiento social.

\begin{tabular}{|c|c|c|c|c|}
\hline & \multicolumn{2}{|c|}{ Respuestas } & \multirow{2}{*}{$\begin{array}{c}\text { Porcentaje } \\
\text { de casos }\end{array}$} \\
\hline & & $\bar{n}$ & Porcentaje & \\
\hline \multirow{5}{*}{$\begin{array}{l}\text { ¿Cuáles han } \\
\text { sido sus } \\
\text { principales } \\
\text { obstáculos en el } \\
\text { proyecto(s) de } \\
\text { emprendimiento } \\
\text { social? }\end{array}$} & Apatía de las personas beneficiadas. & 13 & $12,3 \%$ & $22,4 \%$ \\
\hline & Falta de apoyo de otras personas. & 12 & $11,3 \%$ & $20,7 \%$ \\
\hline & Poco apoyo institucional. & 33 & $31,1 \%$ & $56,9 \%$ \\
\hline & Falta de recursos. & 28 & $26,4 \%$ & $48,3 \%$ \\
\hline & No obtención de resultados. & 20 & $18,9 \%$ & $34,5 \%$ \\
\hline & Total & 106 & $100,0 \%$ & $182,8 \%$ \\
\hline
\end{tabular}

Fuente. Elaboración propia de los autores.

Los resultados obtenidos para esta pregunta se muestran a continuación (Figura 2).

Figura 2. Características del emprendedor social

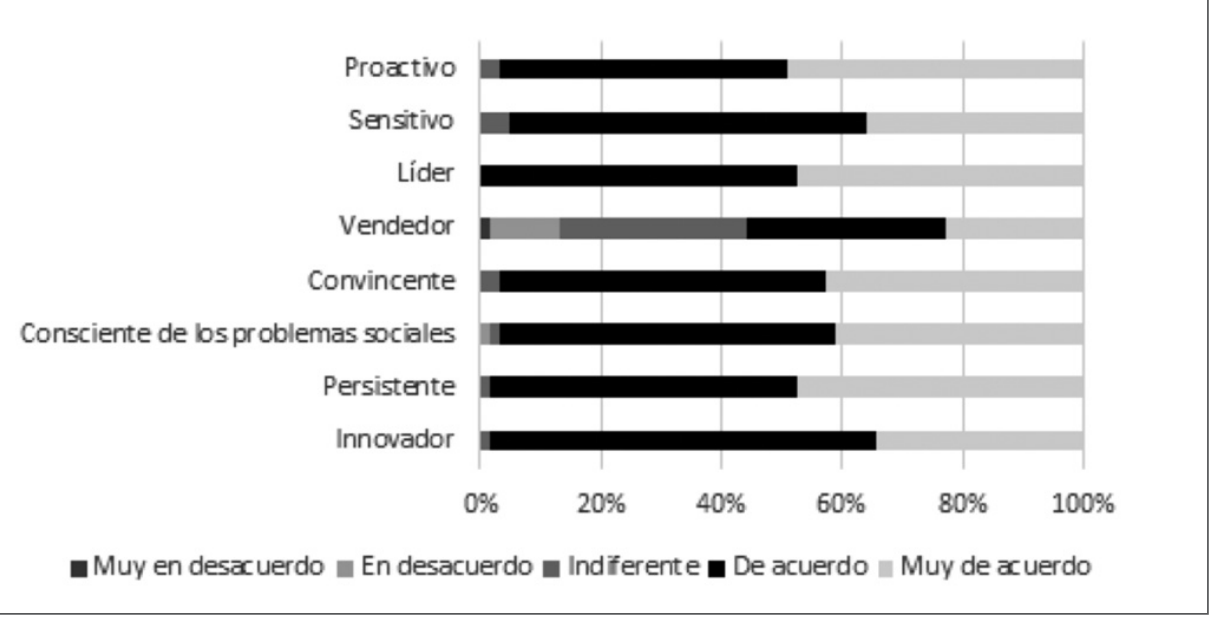

Fuente. Elaboración propia de los autores.

Los encuestados consideran que la mayoría de las características propuestas son importantes para el perfil del emprendedor, pues no existen diferencias significativas entre estas, con excepción de la característica vendedor, en la cual el 44,2 \% la perciben como no relevante dentro de sus competencias.

En la tercera parte del cuestionario, se le pedía al encuestado evaluar la importancia de 13 competencias que debe tener un emprendedor en una escala de 1 a 10, donde diez representa la valoración más alta. Las competencias por evaluar son las siguientes (Figura 3):
- Análisis y solución de problemas.

- Capacidad de adaptación.

- Capacidad de aprender permanentemente.

- Fácil comunicación.

- Habilidad matemática.

- Liderazgo colaborativo.

- Negociación.

- Orientación al cliente.

- Toma de decisiones.

- Trabajo en equipo.

- Capacidad para asumir riesgos y gestión del fracaso.

- Gestión de proyectos.

- Métodos de evaluación de ideas y proyectos. 
Figura 3. Competencias del emprendedor social.

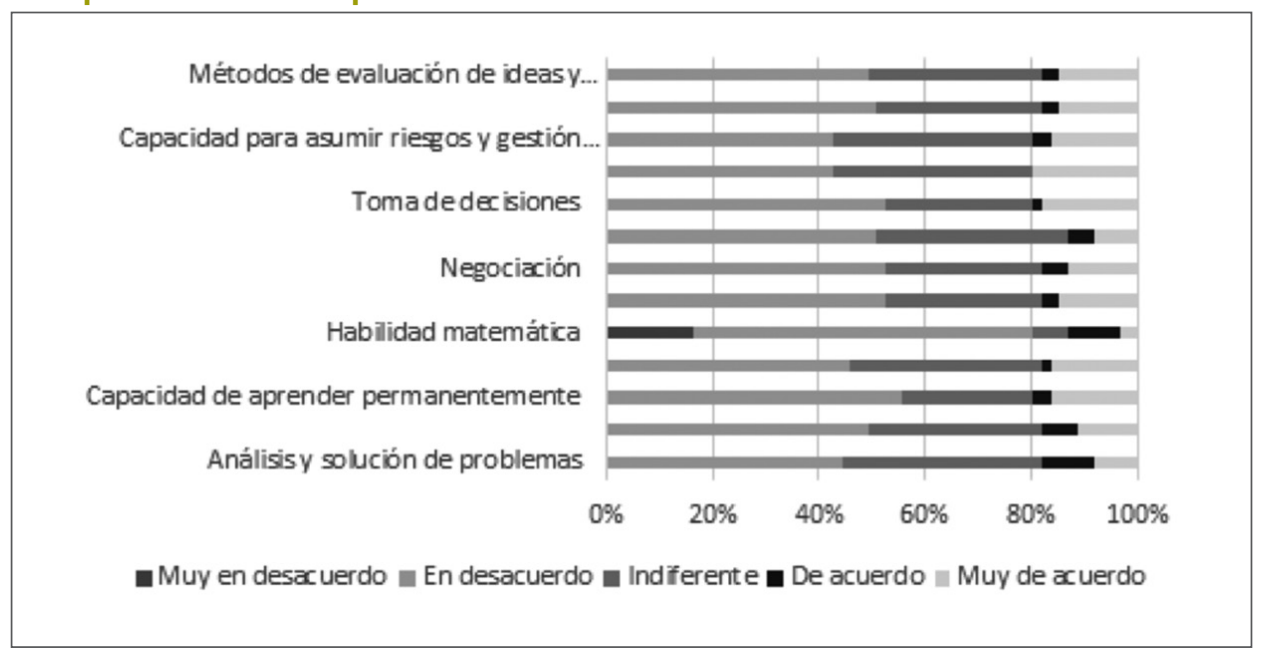

Fuente. Elaboración propia de los autores.

De acuerdo con lo anterior, se muestra que para los emprendedores sociales las competencias no tienen una significación especial. El total de las alternativas planteadas tienen un alto nivel de respuesta en los valores: muy en desacuerdo y en desacuerdo. Sin embargo, sobresale que la menor valoración dada por los emprendedores a alguna de las competencias planteadas corresponde a la habilidad matemática con un $80 \%$ de respuestas dadas en dichos valores. 


\section{Conclusiones}

F rente a los resultados obtenidos, se puede establecer que los emprendedores sociales, que consideran que llevan a cabo proyectos con innovación, no perciben de manera clara que su actividad puede ser un ejercicio empresarial, pues un alto porcentaje lo ven como una actividad con la cual se pueden plantear soluciones a problemas sociales, más con un carácter altruista que con la posibilidad de generar un beneficio económico para el emprendedor.

El análisis nos lleva a concluir que un alto porcentaje de quienes se interesan en proyectos de carácter social, ven en la sociedad problemas a los que ellos pueden brindar soluciones innovadoras, característica a la cual le dan la mayor valoración. En este punto, es tan clara la negación de la actividad empresarial, que la característica menos valorada es la de vendedor, con lo cual se ratifica dicha percepción.

Lo anterior, no sorprende en la misma medida en que uno de los problemas más recurrentes que identifican es la dificultad para la consecución de recursos, dado que la competencia de vendedor involucra, en primera instancia, la capacidad para vender la idea del proyecto.

Resultan consistentes los resultados cuando el reconocimiento de la comunidad es catalogado como el segundo logro más importante logro, y las dos menores menciones, en cuanto a los obstáculos, son precisamente la apatía de los beneficiarios y la falta de apoyo de otras personas. Por el contrario, el emprendedor sí siente que hay ausencia de apoyo institucional, entendido como la baja participación de las instituciones gubernamentales en sus iniciativas de servicio a la comunidad.

Por último, resulta importante resaltar el poco valor que dan a los conocimientos que han adquirido en sus procesos de formación, bien sea en el colegio o en la universidad; más allá de los conocimientos, representados en la baja calificación dada, por ejemplo, a la habilidad matemática, dan mayor valor a otras competencias relacionadas con el desarrollo social y las habilidades para la gestión. 


\section{Referencias bibliográficas}

Abreu Quintero, J. L. (2011). Innovación social: conceptos y etapas. Daena: International Journal of Good Conscience, 6(2), 134-148.

Rey, N., y Tancredi, F. (2010). De la innovación social a la política pública. Santiago de Chile: CEPAL.

Consejo Europeo de Lisboa, (2000). Conclusiones de la Presidencia. Recuperado de: http://www.europarl.europa.eu/ summits/lis1_es.htm

Christensen, M. A. (1994). The identification of Business Starters using attitude. Calgary: University of Calgary Press.

Dees, J. G. (1998). The Meaning of Social Entrepreneurship. Stanford University: Draft Report for the Kauffman Center for Entrepreneurial Leadership.

Dess, G., Lumpkin, G., \& Eisner, A. (2010). Strategic management: Text and cases. Columbus. USA: McGraw-Hill//rwin.

Enciso-Congote, J. D. (2010). El emprendimiento y el bien común. Educación y educadores, 13(1), 63-76.

Gilomen, H. (2006). Resultados deseados: una vida de éxito y un buen funcionamiento de la sociedad. En Rychen, D. Salganik L. (Ed.). Las competencias clave para el bienestar personal, social y económico, pp. 127-147. Málaga, España: Editorial Aljibe.

Martínez, R. (2011). Politicas públicas e innovación social. Marcos conceptuales y efectos en la formulación de las políticas. Trabajo final (Master Pensar y Gobernar las Sociedades Complejas). Barcelona: Universidad Autónoma de Barcelona.

Murphy, P. J., \& Coombes, S. M. (2009). A model of social entrepreneurial discovery. Journal of business ethics, 87(3), 325-336.

Murray, R., Caulier-Grice, J., y G. Mulgan (2010). The Open Book of Social Innovation. Recuperado de: http://www. youngfoundation.org/files/images/Open_ Book_of_Social_Innovation.pdf

Olamendi, G. (2005). Cómo crear una empresa y triunfar en el intento. Santiago de Chile: Limusa.

Ridley-Duff, R. (2008). Social enterprise as a socially rational business. International Journal of Entrepreneurial Behavior \& Research, 14(5), 291-312.

Sastre-Castillo, M. A., Peris-Ortiz, M., y DanvilaDel Valle, I. (2015). What Is Different about the Profile of the Social Entrepreneur? Nonprofit Management and Leadership, 25(4): 349-369. doi: 10.1002/nml.21138.

Schumpeter, J. (1911). Teoría del desenvolvimiento económico. Quinta reimpresión. México: Fondo de Cultura Económica, México, 1978.

Vázquez, Y. A. (2001). Educación basada en competencias. Educar: revista de educación/ nueva época, 16, pp.1-29.

World Bank Group. (2016). Global Monitoring Report 2015/2016: Development Goals in an Era of Demographic Change. Washington, DC: World Bank. doi: 10.1596/978-14648-0669-8. 